Case Report

\title{
A Case of an Abdominal Aortic Dissection in a Hemodynamically Stable Marfan Syndrome Patient Presenting without Pain
}

\author{
Hussein Al-Mohamad $\mathbb{D}^{,}{ }^{1}$ Kara Stout $\mathbb{D}^{\mathbb{C}}{ }^{2}$ Taryn Bolling, ${ }^{2}$ and Ronald Walsh $\mathbb{D}^{1}$ \\ ${ }^{1}$ Department of Cardiology, Largo Medical Center, Largo, FL, USA \\ ${ }^{2}$ Department of Internal Medicine, Largo Medical Center, Largo, FL, USA \\ Correspondence should be addressed to Hussein Al-Mohamad; halmohamad@vcom.vt.edu
}

Received 9 June 2019; Accepted 2 November 2019; Published 25 February 2020

Academic Editor: Assad Movahed

Copyright (C) 2020 Hussein Al-Mohamad et al. This is an open access article distributed under the Creative Commons Attribution License, which permits unrestricted use, distribution, and reproduction in any medium, provided the original work is properly cited.

\begin{abstract}
Introduction. Marfan syndrome (MFS) is a rare connective tissue disorder attributed to a defect in the fibrillin-1 gene. Aortic aneurysms and dissection are common causes of morbidity and mortality in Marfan syndrome. Case Report. A 43-year-old female with a history of MFS and a $4.0 \mathrm{~cm}$ dilated ascending aorta presented to her cardiologist reporting that since a C-section two years prior, the left side of her abdomen painlessly protruded when standing. An outpatient CT scan of the abdomen/pelvis noted a $5.5 \mathrm{~cm}$ abdominal aortic dissection, and she was directed to the hospital. Repeat CT scan of the abdomen/pelvis revealed a $5.6 \mathrm{~cm}$ dissecting aneurysm of the infrarenal abdominal aorta. The patient was admitted to the ICU and started on a nitroglycerin drip to maintain systolic blood pressure less than $110 \mathrm{mmHg}$. The patient underwent repair of her abdominal aortic dissection via a retroperitoneal approach, and she tolerated the procedure well. She was started on metoprolol tartrate $12.5 \mathrm{mg}$ BID and aspirin $81 \mathrm{mg}$ postoperatively. She was safely discharged with follow-up care. Conclusion. This case stresses the importance of having a low threshold to obtain imaging in a MFS patient with protruding abdomen, even though the patient may not have pain and be hemodynamically stable.
\end{abstract}

\section{Introduction}

Marfan syndrome (MFS) is a rare connective tissue disorder with an estimated incidence of about 1 in 5,000 persons in the United States; it is generally inherited in an autosomal dominant pattern $[1,2]$. The pathogenesis involves a defect in the fibrillin-1 (FBN-1) gene located on chromosome 15 [2-4]. The role of FBN-1 is to provide structural support to tissues and to regulate elastogenesis [5]. Defects in this gene can lead to mitral valve prolapse (MVP), joint laxity, and ectopia lentis [5]. More serious clinical findings include aortic root dilatation and thoracoabdominal aortic aneurysms and dissections [2]. Annual echocardiograms are recommended for patients with known MFS to monitor for aortic root dilatation [6].

Several cases have been reported about abdominal aortic aneurysms, dissections, and ruptures [2, 3, 7-9]. In review of the literature, it is rare to find a case of a hemodynamically stable and fairly asymptomatic abdominal aortic dissection in a patient with MFS. We present such a case to increase awareness about this rare, yet severe complication.

\section{Case Report}

We present a case of a 43-year-old nonsmoking female with a past medical history of MFS, MVP, and a $4.0 \mathrm{~cm}$ dilated ascending aorta who presented to her cardiologist after being diagnosed with an abdominal aortic dissection by her OB-GYN. The patient noted that since a C-section 2 years prior, the left side of her abdomen protruded more when standing compared to the right. She denied associated abdominal, chest, or back pain. The patient was told that she may have a hernia in the past.

Because she was concerned about the asymmetry of her abdomen, she went to a new OB-GYN who ordered an abdominal CT scan of the abdomen/pelvis with IV contrast which showed a $5.5 \mathrm{~cm}$ abdominal aortic dissection. The patient was reluctant to go to the hospital until she saw her 


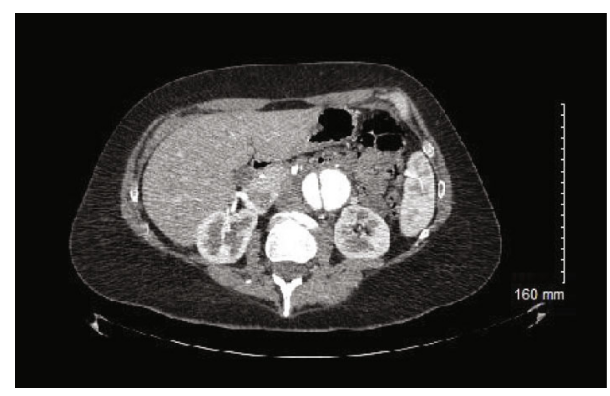

FIGURE 1: CT scan of the abdomen/pelvis with IV contrast demonstrating dissection of the abdominal aorta.

cardiologist a few days later, who recommended hospitalization and a consult for cardiothoracic surgery.

Interestingly, when the patient was seen in the hospital, she again denied chest pain, back pain, abdominal pain, dyspnea, nausea, vomiting, and dizziness. She only mentioned that her left side of the abdomen protrudes more than the right side when standing. On physical exam, patient had several features typical to MFS: she was tall, had a high arched palate, arachnodactyly, pectus excavatum, and her arm span was greater than her height. Her abdomen was soft, nontender, nondistended, had normal bowel sounds, and no pulsatile mass was appreciated. However, when the patient stood up, it was clear that the left side of her abdomen was bulging out in comparison to the right. A repeat CT scan of the abdomen/pelvis revealed a dissecting aneurysm of the infrarenal abdominal aorta measuring up to $5.6 \mathrm{~cm}$ (Figure 1); the false lumen supplied the left common iliac artery and the true lumen supplied the right common iliac artery. There was no evidence of thoracic aortic dissection.

The patient was admitted to the ICU and started on a nitroglycerin drip to maintain a systolic blood pressure less than $110 \mathrm{mmHg}$. Cardiothoracic surgery was consulted and recommended the patient to undergo open surgical repair of the abdominal aortic dissection. In order to risk stratify the patient prior to the procedure, the patient underwent a regadenoson nuclear medicine stress test, which was low risk for ischemia. The patient underwent successful repair of her abdominal aortic dissection via a minimally invasive open retroperitoneal approach. She tolerated the procedure well without postoperative complications.

The patient came into the hospital with no home medications. As she has reported in the past, she was tried on nebivolol but had failed it due to syncope. Given this, metoprolol tartrate $12.5 \mathrm{mg}$ PO BID was started cautiously to provide long-term benefits for the patient's abdominal and thoracic aorta. The patient tolerated this medication well and was safely discharged home with close cardiovascular follow-up.

\section{Discussion}

Aortic root disease leading to aneurysmal dilation, regurgitation, and dissection is the most common cause of morbidity and mortality in MFS [10]. Aortic aneurysms and dissections arise due to the fragility of the aortic tissues, which are responsible for the rise of cystic medial necrosis [11].
Class I screening guidelines by the American College of Cardiology (ACC) include an echocardiogram at the time of diagnosis of MFS to determine the diameter of the aortic root and ascending aorta. Repeat imaging is recommended at six months and then annually to follow the rate of enlargement. If the aorta is greater than $4.5 \mathrm{~cm}$ or has significantly changed from baseline, more frequent screening is recommended [12].

Pregnancy in women with MFS can be challenging and is associated with an increased risk of aortic dissection. Recommendations include preconception counseling, TTE, and initiation of a protective beta blocker to slow the growth of the aortic root [13]. Serial TTE's are performed every 4-12 weeks to monitor aortic size, and C-section is recommended for aortic root dilation over $4 \mathrm{~cm} \mathrm{[13].} \mathrm{For} \mathrm{aortic} \mathrm{root} \mathrm{dilatations}$ over $4.5 \mathrm{~cm}$, prophylactic aortic root and ascending aorta replacement is recommended prior to pregnancy [13]; but, even after successful root replacement, these patients remain at high risk for future events [14]. In our case, the patient followed the pregnancy guidelines and delivered her child via a $\mathrm{C}$-section.

While MFS commonly leads to aortic root aneurysm and descending thoracic aortic aneurysms, the presence of an abdominal aortic aneurysm (AAA) and abdominal aortic dissection is rarely reported [3, 4, 7-9]. Up to $20 \%$ of patients with infrarenal AAAs have a family history of AAAs, suggesting there is a likely inherited component. Routine spiral thinslice CT angiography or magnetic resonance angiography imaging of the entire aorta is recommended if there is enlargement of the descending thoracic aorta and abdominal aorta or if there is aortic dissection $[6,15]$.

Strategies to combat the increased risk of dissection include life-long $\beta$-blockade and elective aortic root replacement at $5 \mathrm{~cm}$ [2]. In addition, low-dose aspirin therapy helps to reduce major coronary events, cardiovascular-related mortality, and ischemic stroke risk [16]. Blood pressure management with a beta blocker up titrated to a goal heart rate less than 100 beats per minute with exercise is initiated to reduce cardiovascular mortality [15]. In our case, the patient was started on metoprolol tartrate and low-dose aspirin to maximize risk reduction for future events.

A decision to repair an AAA is also not MFS specific. It is based on size greater than or equal to $5.5 \mathrm{~cm}$ in males, $5 \mathrm{~cm}$ in females, or expansion of more than $5 \mathrm{~mm}$ in 6 months or $1 \mathrm{~cm}$ over one year [16]. Risk factors for rupture include advanced age, male sex, Caucasians, smoking, other large vessel aneurysms, and atherosclerosis $[16,17]$.

The decision to repair a dissection depends on the type of dissection but is also not specific to MFS. Stanford Type A dissections are a surgical emergency [18]. For Stanford Type $\mathrm{B}$ dissections, medical treatments are initially attempted with esmolol or labetalol to keep systolic blood pressure between 100 and $120 \mathrm{mmHg}$ and nitroprusside for vasodilation [18]. In Type B dissections, indications for surgery include the evidence of end-organ malperfusion, refractory pain, a rapidly expanding false lumen, impending or frank rupture, or a chronic aneurysmal dilation with a diameter of greater than or equal to $5 \mathrm{~cm}$ [18]. In our case, the patient met the final criteria of chronic aneurysmal dilation over $5 \mathrm{~cm}$. Nitroprusside 
was used for both blood pressure management and vasodilation. Perioperative management includes an assessment for underlying coronary artery disease and ischemia if needed [6]. In this case, our patient underwent a regadenoson nuclear medicine stress test prior to her surgical procedure.

In general, endovascular techniques were not initially intended for thoracic and abdominal aorta pathology in patients with connective tissue diseases [14, 19]. Patients specifically with MFS were not included in initial endograft studies due to the fragile nature of the marfanoid aorta and concern for the predictive ability of the long-term radial force of the endograph to provide a seal without adversely damaging the vessel $[14,19]$. Furthermore, as patients are typically young and with unclear long-term durability of current stent grafts, these should be avoided [14]. The European Society of Vascular Surgery guidelines recommend an open surgical procedure, with considerations for EVAR only if the patient is deemed too high of a risk for the open intervention [14] or if EVAR is a life-saving bridging procedure until definitive open repair can be safely performed [19]. In accordance, a minimally invasive retroperitoneal approach for open AAA repair was performed for our patient, and she tolerated the procedure without major complications.

\section{Conclusion}

In conclusion, this case stresses the importance of having a low threshold to obtain imaging in a MFS patient with bulging or protruding abdomen, even though, the patient may not have pain and be hemodynamically stable.

\section{Consent}

The patient provided informed consent.

\section{Disclosure}

The views expressed in this publication represent those of the author(s) and do not necessarily represent the official views of HCA Healthcare or any of its affiliated entities.

\section{Conflicts of Interest}

The authors declare no financial interest nor any other conflict of interest.

\section{Acknowledgments}

We would like to thank Dr. Furqan Haq for his assistance in reviewing and helping to prepare this manuscript. Secondly, we would like to thank Dr. Christopher Stone of cardiothoracic surgery for his clinical input and surgical expertise. This research was supported (in whole or in part) by HCA Healthcare and/or an HCA Healthcare-Affiliated Entity.

\section{References}

[1] S. Westaby, "Aortic dissection in Marfan's syndrome," The Annals of Thoracic Surgery, vol. 67, no. 6, pp. 1861-1863, 1999.
[2] B. Wolfgarten, I. Kruger, and M. Gawenda, "Rare manifestation of abdominal aortic aneurysm and popliteal aneurysm in a patient with Marfan's Syndrome," Vascular Surgery, vol. 35, no. 1, pp. 81-84, 2001.

[3] A. Verstraeten, M. Alaerts, L. van Laer, and B. Loeys, "Marfan syndrome and related disorders: 25 years of gene discovery," Human Mutation, vol. 37, no. 6, pp. 524-531, 2016.

[4] T. Takayama, T. Miyata, and H. Nagawa, "True abdominal aortic aneurysm in Marfan syndrome," Journal of Vascular Surgery, vol. 49, no. 5, pp. 1162-1165, 2009.

[5] B. L. Loeys, H. C. Dietz, A. C. Braverman et al., "The revised Ghent nosology for the Marfan syndrome," Journal of Medical Genetics, vol. 47, no. 7, pp. 476-485, 2010.

[6] D. M. Milewicz, H. C. Dietz, and D. C. Miller, "Treatment of aortic disease in patients with Marfan syndrome," Circulation, vol. 111, no. 11, pp. e150-e157, 2005.

[7] T. Hagerty, P. Geraghty, and A. C. Braverman, "Abstract 12688: abdominal aortic aneurysm in Marfan syndrome: time for screening guidelines?," Circulation, vol. 132, Supplement 3, 2015.

[8] K. Lafferty, L. McLean, J. Salisbury, and L. T. Cotton, "Ruptured abdominal aortic aneurysm in Marfan's syndrome," Postgraduate Medical Journal, vol. 63, no. 742, pp. 685-687, 1987.

[9] S.-M. Yuan and H. Jing, "Cystic medial necrosis: pathological findings and clinical implications," Brazilian Journal of Cardiovascular Surgery, vol. 26, no. 1, pp. 107-115, 2011.

[10] J. N. Adams and R. J. Trent, “Aortic complications of Marfan's syndrome," The Lancet, vol. 352, no. 9142, pp. 1722-1723, 1998.

[11] D. P. Zipes, P. Libby, B. O. Bonow, D. L. Mann, G. F. Tomaselli, and E. Braunwald, Braunwald's Heart Disease: A Textbook of Cardiovascular Medicine, Elsevier, Philadelphia, PA, USA, Eleventh edition, 2019.

[12] L. F. Hiratzka, G. L. Bakris, J. A. Beckman et al., ACC/AHA pocket guideline: guidelines for the diagnosis and Management of Patients with thoracic aortic disease, The American College of Cardiology Foundation and the American Heart Association, 2010, https://www.acc.org/ /media/clinical/pdf-files/ approvedpdfs/2015/01/13/11/13/thoracic\%20aortic\% 20disease\%202010\%20pocket\%20guide.pdf.

[13] S. Goland and U. Elkayam, "Pregnancy and Marfan syndrome," Annals of Cardiothoracic Surgery, vol. 6, no. 6, pp. 642-653, 2017.

[14] L. G. Svensson, N. T. Kouchoukos, D. C. Miller et al., "Expert consensus document on the treatment of descending thoracic aortic disease using endovascular stent-grafts," The Annals of Thoracic Surgery, vol. 85, no. 1, Supplement, pp. S1-S41, 2008.

[15] Marfan Foundation, "Overview of cardiac management of Marfan syndrome,” 2014, https://www.marfan.org/resource/ fact-sheet/cardiac-management-marfan-syndrome\# .XbtVtEVKhE4.

[16] F. L. Moll, J. T. Powell, G. Fraedrich et al., "Management of abdominal aortic aneurysms clinical practice guidelines of the European society for vascular surgery," European Journal of Vascular and Endovascular Surgery, vol. 41, no. 1, pp. S1-S58, 2011.

[17] E. L. Chaikof, R. L. Dalman, M. K. Eskandari et al., "The Society for Vascular Surgery practice guidelines on the care of patients with an abdominal aortic aneurysm," Journal of Vascular Surgery, vol. 67, no. 1, pp. 2-77.e2, 2018. 
[18] R. Erbel, V. Aboyans, C. Boileau et al., “2014 ESC guidelines on the diagnosis and treatment of aortic diseases: document covering acute and chronic aortic diseases of the thoracic and abdominal aorta of the adult: the Task Force for the Diagnosis and Treatment of Aortic Diseases of the European Society of Cardiology (ESC)," European Heart Journal, vol. 35, no. 41, pp. 2873-2926, 2014.

[19] D. Böckler, K. Meisenbacher, A. S. Peters, C. Grond-Ginsbach, and M. S. Bischoff, "Endovascular treatment of genetically linked aortic diseases," Gefasschirurgie, vol. 22, no. S1, Supplement 1, pp. 1-7, 2017. 